\title{
Hierarchical Model for the Analysis of Scattering Data of Complex Materials
}

\author{
AKINOLA OYEDELE,${ }^{1}$ NICHOLAS W. MCNUTT, ${ }^{2}$ ORLANDO RIOS, ${ }^{3}$ \\ and DAVID J. KEFFER (1D ${ }^{2,4}$
}

1.-Bredesen Center for Interdisciplinary Research and Graduate Education, University of Tennessee, Knoxville, TN 37996, USA. 2.-Department of Materials Science and Engineering, University of Tennessee, Knoxville, TN 37996, USA. 3.-Materials Science \& Technology Division, Oak Ridge National Laboratory, Oak Ridge, TN 37830-8026, USA. 4.-e-mail: dkeffer@utk.edu

\begin{abstract}
Interpreting the results of scattering data for complex materials with a hierarchical structure in which at least one phase is amorphous presents a significant challenge. Often the interpretation relies on the use of large-scale molecular dynamics (MD) simulations, in which a structure is hypothesized and from which a radial distribution function (RDF) can be extracted and directly compared against an experimental RDF. This computationally intensive approach presents a bottleneck in the efficient characterization of the atomic structure of new materials. Here, we propose and demonstrate an approach for a hierarchical decomposition of the RDF in which MD simulations are replaced by a combination of tractable models and theory at the atomic scale and the mesoscale, which when combined yield the RDF. We apply the procedure to a carbon composite, in which graphitic nanocrystallites are distributed in an amorphous domain. We compare the model with the RDF from both MD simulation and neutron scattering data. This procedure is applicable for understanding the fundamental processing-structure-property relationships in complex magnetic materials.
\end{abstract}

\section{INTRODUCTION}

Modern material discovery efforts are predicated on the development of predictive structure/property relationships. These relationships must invoke structural descriptors that contain information relevant to the properties of interest. Often these descriptors are measures of the local environment at the atomic scale and the mesoscale. Typically, the representation available of the structure of a material comes from x-ray or neutron scattering experiments in the form of the structural function, $S(Q)$ or equivalently its Fourier transform, the radial distribution function (RDF). For crystalline materials, the transformation of this data-lean description to a data-rich representation, (e.g., the atomic coordinates and lattice parameters of the unit cell) is routinely accomplished via existing software. ${ }^{1,2}$ However, for complex materials, which are highly disordered, amorphous, and/or hierarchically structured, neither the interpretation of the RDF nor its transformation to a data-rich format is straightforward. On-going efforts exist to develop a general approach to extracting information regarding the structure of complex materials via scattering. ${ }^{3,4}$

One approach currently used involves hypothesizing the structure with a sufficient number of atoms to capture the structure on length scales of interest. For large systems, molecular dynamics (MD) simulation is invoked to equilibrate the structure. Once equilibrated, an RDF can be generated from the simulation and compared directly to the experimental RDF. If the agreement between simulation and experiment is poor, another iteration of the simulations must be performed. Examples can be found for amorphous iron ${ }^{5}$ and for carbon composites, in which graphitic nanoparticles are dispersed within a disordered amorphous carbon matrix. ${ }^{6-8}$ This approach is computationally and labor intensive.

This article introduces an alternative approach to the interpretation of the scattering data of complex materials, which removes this computational 
bottleneck and has the potential to be automated in a manner analogous to software targeted at crystalline materials. This approach embraces the hierarchical structure of complex materials by introducing a hierarchical decomposition of the RDF. If each component of an RDF can be described in terms of a well-established model or theory at the atomic scale or the mesoscale, then a satisfactorily accurate understanding of the structure contained within the scattering data can be quickly generated. Such an approach has the potential to speed up the rate of material discovery in many fields. For example, non-rare-earth magnetic materials, ${ }^{9}$ hybrid perovskites, ${ }^{10}$ conducting polymers, ${ }^{11}$ solid state electrolytes, ${ }^{12}$ carbon fibers, ${ }^{13}$ and memory polymers ${ }^{14}$ are complex materials composed of both amorphous and crystalline domains.

A hierarchical structure in which crystalline nanoparticles, displaying hard magnetism, are dispersed uniformly within an amorphous ferromagnetic matrix, displaying soft magnetism has been discovered in melt-spun ribbons of rare-earth-free $\mathrm{Hf}_{2} \mathrm{Co}_{11} \mathrm{~B} .{ }^{9}$ This composite material exhibited comparable ferromagnetic performance to the best existing materials. The ability to predictively understand how processing conditions modify the structure and, in turn, their impact on magnet performance would address fundamental scientific questions regarding emergent properties in hierarchically structured materials and specifically deliver new permanent magnets.

In this work, we provide the first demonstration of this hierarchical decomposition for carbon composites, based on pyrolyzed lignin. The model RDF is compared to MD simulation on a component-bycomponent basis to demonstrate that this approach is a physics-based rather than a fitting algorithm. The model RDF is also compared to the experimentally determined scattering data from the Spallation Neutron Source (SNS). Since the crystalline/amorphous composite magnets are mesoscopic isomorphs of the carbon composites, the developed tool can be directly extended to understand the basis of processing-structure-property relationships in nonrare-earth magnets.

\section{NATURE OF THE DECOMPOSITION}

Because the RDF describes correlations between pairs of atoms, at the first level of decomposition, a general composite can be formulated in terms of interactions between atoms located within the same phase (intraphase) and those in different phases (interphase). ${ }^{15}$ In the example shown in Fig. 1a, the carbon composite is composed of two phases, the first consisting of crystalline (graphitic) nanoparticles shown in gray and the second consisting of amorphous carbon, represented as fragments of graphene shown in blue. As illustrated, both phases need not be contiguous and the model is directly generalizable to materials with more than two
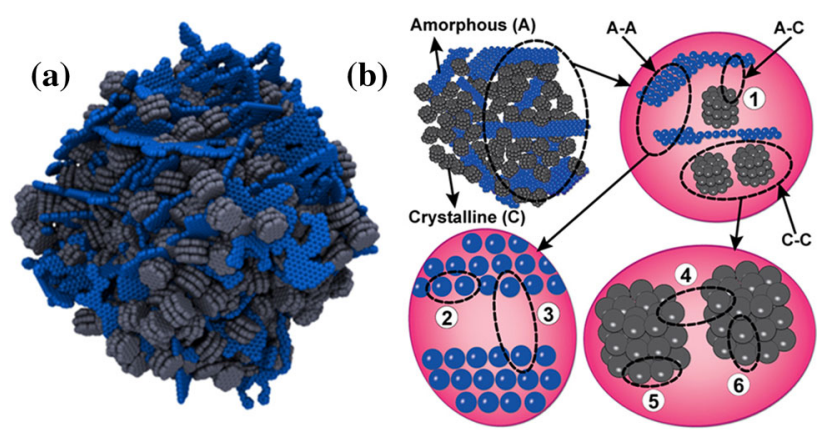

(c)

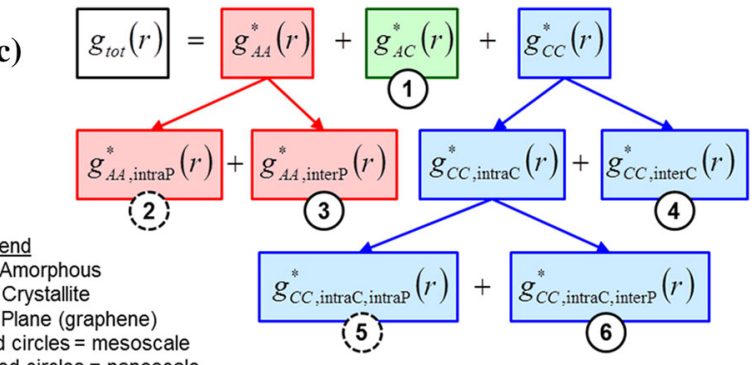

solid circles $=$ mesoscale

Fig. 1. Model structure and the general formulation of its decomposition of a carbon composite. (a) Structural model of a lignin-derived carbon composite used as a battery anode material consisting of crystalline graphitic nanoparticles (in gray) distributed in a matrix of amorphous carbon (in blue). The crystallite size, $r_{\mathrm{c}}=5 \AA$, composite density, $\rho=1.94 \mathrm{~g} / \mathrm{cm}^{3}$ and crystalline volume fraction, $\phi_{\mathrm{c}}=0.9^{7}$ (b) Schematic of the hierarchical decomposition of the model structure. (c) The resulting decomposition equation of the RDF.

phases and with diffuse boundaries. The first level of decomposition, as shown on the first line of Fig. 1c, contains three terms: an amorphous-amorphous (AA), amorphous-crystalline (AC), and a crystalline-crystalline (CC) component.

The ability to further decompose the model depends on the ability to identify smaller structural units in each phase. For an interphase component, such as AC, there is likely no further decomposition. For intraphase components, there are several options, two of which are illustrated in this example. In the amorphous phase, we consider the structural unit to be a fragment of graphene sheets, a choice motivated by the graphitization induced by the pyrolysis process and ultimately validated by comparison of the simulated RDF with experiment. In this case, the AA term is decomposed at the second level into interplanar (AA,interP) and intraplanar (AA,intraP) terms. For phases composed of nanoparticles such as the crystalline phase, the nanoparticle is a structural unit. Thus, the CC term is decomposed at the second level into intercrystallite (CC,interC) and intracrystallite (CC,intraC) terms. Each nanoparticle has an internal structure that can be further decomposed. Since the particle is graphitic, a layered crystal, the CC,intraC term can be decomposed into interplanar (CC,intraC,interP) and intraplanar (CC,intraC,intraP) terms. For other nanoparticles with negligible internal degrees 
of freedom, further decomposition of the nanoparticle may not be necessary. In this example, the six components pulled from the first, second, and third levels of decomposition are illustrated graphically in Fig. 1b, whose numbering correspond to the analogously numbered terms in Fig. 1c.

The goal in this decomposition is to create a set of components for which an $\mathrm{RDF}$ can be readily evaluated by theory without resorting to MD simulation. However, to demonstrate that the process is not simply an exercise in curve fitting, we must establish a physical basis for each component of the RDF. To do so, we require a decomposition based on MD as a validation of the procedure. The example in this work illustrates the simplicity and broad applicability of the decomposition approach. In the case of a carbon composite, six components are identified, pulled from the first, second, and third levels of decomposition. These components are illustrated graphically in Fig. 1b. The numbering of the components in Fig. $1 \mathrm{~b}$ corresponds to the analogously numbered terms in Fig. 1c.

\section{Justification of the Decomposition}

Since only the total RDF is available experimentally, the advantage of a decomposition has not been apparent. However, for this carbon composite example, a full MD simulation analysis of a suite of composites was performed. ${ }^{7}$ From simulation, the decomposition can be easily performed since the origin of each pair correlation can be readily identified as one of the six components in Fig. 1. The decomposition of the RDF not only provides a much clearer understanding of the origin of each feature in the total RDF, but it also provides additional insight into the structure captured in the RDF. In Fig. 2, the six components of the RDF as obtained from MD simulation are plotted.

With the advantage of seeing the utility of this decomposition, we were motivated to find an approach that can reproduce it without resorting to $\mathrm{MD}$ simulations.

\section{Evaluation of the Decomposition}

Each component of the RDF can be approximated in a straightforward manner at either the atomicscale or the mesoscale level. The nature of each decomposition is described as follows.

\section{Amorphous-Crystalline (AC) Component}

This component represents an interphase contribution between pairs of atom in the amorphous and crystalline phase. Here a mesoscale, mean-field theory is adopted that neglects the atomistic structure of the material, replacing it with a continuum mean-field density. The evaluation of the AC component requires the RDF describing the interaction of a single nanoparticle in an infinite amorphous matrix followed by an averaging over the distribution of nanoparticles. The single-particle $\mathrm{RDF}$ is generated through a four-step process that is used repeatedly in this work. First, a joint probability distribution is formulated over the six independent coordinates of the two atoms in the pair. Since the coordinates are independent, the joint probability distribution is the product of the single-variable probability distributions. Second, the radial argument $r$ of the RDF is defined as a function of the independent coordinates in the system. Third, the cumulative distribution function is obtained in terms of the single variable of interest, $r$, by analytically integrating over all the independent variables across the volumes of interest, e.g., a sphere for a nanoparticle. Fourth, the differentiation of the cumulative probability distribution function, $\mathrm{F}(r)$, with respect to $r$ yields the probability distribution function, $\mathrm{f}(r)$, which can then be normalized to give the component RDF, $\mathrm{g}(r)$.

In the evaluation of the $\mathrm{AC}$ component, there are two parameters in the model, the atom size, $\sigma$, and the radius of the crystallite, $r_{\mathrm{c}}$, whose values were $2.8 \AA$ and $5 \AA$, respectively, as determined from structural characterization experiments such as the transmission electron microscope. We assumed that the nanoparticles were distributed uniformly throughout the material, although the approach readily accommodates other distributions. The result of the model for the AC component is shown in Fig. 2a, along with the RDF obtained from MD simulation. ${ }^{7}$ The AC component of the RDF rises steeply at $\sigma$ and then quickly plateaus. The simulation RDF shows some additional features at short distances. However, it is unclear whether these features are artifacts from a small system size $(70,000$ atoms in this simulation) that would disappear through the averaging present in larger systems. There is no experiment to compare with for a single component of the RDF. Comparison with experiment must be delayed until all components are generated.

\section{Amorphous-Amorphous, Intraplanar (AA,intraP) Component}

Based on an understanding of the pyrolysis process of lignin, the amorphous phase of this carbon composite was modeled as randomly oriented fragments of graphene. At low crystalline volume fraction, the amorphous phase is a continuous matrix. At high crystalline volume fraction (as is the case in this example), the amorphous phase fills interstitial spaces between crystallites. Although in the simulation, the amorphous phase is composed of a distribution of fragment sizes, in the model, we assume a single, circular fragment. Thus, there is one geometric parameter in this component, the characteristic radius of the graphene planes in the amorphous domain, $r_{\mathrm{a}}$, which is $2.5 \AA$. To evaluate this term, we took the known crystal structure of graphene and cut out a circle of radius $r_{\mathrm{a}}$ and explicitly evaluated the $\mathrm{RDF}$, a trivial 

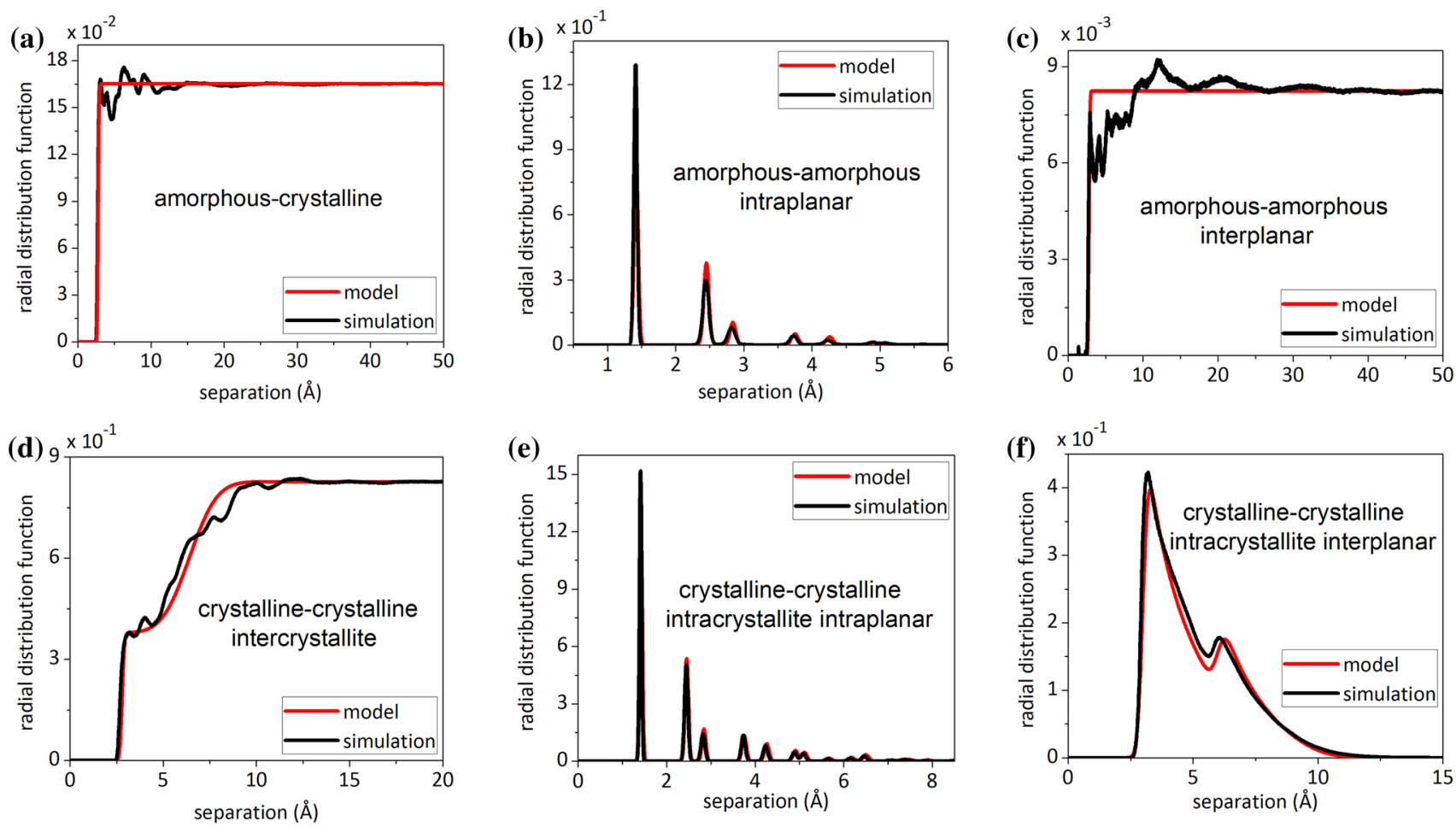

Fig. 2. Comparison of the component RDFs from model and MD simulation: (a) AC component (b) AA, intraP component, (c) AA,interP component, (d) CC, interC component, (e) CC, intraC, intraP component, and (f) CC, intraC, interP component.

exercise given that such a fragment contains only 10 $\mathrm{C}$ atoms. We then broadened each peak with a Gaussian distribution representing thermal noise. The variance of this Gaussian represents a second parameter for this component, which captures both temperature and the force constant of the C-C bond within a graphene plane. As shown in Fig. 2b, the AA,intraP component of the RDF from model and simulation agree very well.

\section{Amorphous-Amorphous, Interplanar (AA,interP) Component}

In general, we have very little information regarding the irregular shape of the amorphous domain or the organization of graphene fragments within it. Therefore, we rely on a low-order approximation for this component, namely, the mean-field approximation again. This approximation requires the atom size, $\sigma$, already introduced and a second (and final) Gaussian distribution variance. As shown in Fig. 2c, the AA,interP rises and then reaches a plateau at 0.008 . The model agrees qualitatively with the simulation. Again, the discrepancy lies in features at short distances, which may be artifacts due to small simulation sizes.

\section{Crystalline-Crystalline, Intercrystallite (CC,interC) Component}

The CC,interC component represents contributions from atoms in different crystallites. As was the case in the $\mathrm{AC}$ component, the same mesoscale model of spherical nanoparticles without atomistic detail is invoked. Following the same procedure, we analytically derived the RDF for a pair of spheres, separated by distance, $d$. We then assumed the same uniform distribution of spheres beginning at separations that avoided particle overlap. This resulted in a curve with the general, smooth shape of a complementary error function. This approach worked very well for systems with low-crystallite volume fractions. However, for high values of $\phi_{\mathrm{c}}(0.9$ in this example), we observed a more complicated structure in the RDF. In fact, at high $\phi_{c}$, two features are observed, the sharp rise due to an atomic interface as shown in Fig. $2 \mathrm{a}$ and $\mathrm{c}$ and a much slower rise over the particle size. Thus, the $\mathrm{CC}$,interC was captured in two effects as shown in Fig. 2d, in which a steep rise due to atomic interactions between two crystallites pressed against each other eventually leads to a more general rise reflecting the shape of the nanoparticle.

\section{Crystalline-Crystalline, Intracrystallite, Intrapla- nar (CC,intraC,intraP) Component}

The interaction between two carbon atoms within the same plane of a crystallite is treated in an exactly analogous way as was the intraplanar interaction in the amorphous phase, through explicit counting of pairs from the graphene crystal structure for a plane of radius, $r_{\mathrm{c}}$, which is again trivial as a plane of radius $5 \AA$ contains $31 \mathrm{C}$ atoms. The assumption that all planes in the crystallite are 
the same size does not correspond to a spherical nanoparticle but to a cylindrical nanoparticle. This is a nonessential simplifying assumption used in this analysis. The value of $5 \AA$ corresponds to the radius of the nanocrystallites used in the simulation. The peaks are broadened with the same Gaussian distribution used in the amorphous phase, resulting in no additional structural parameters for this component. As shown in Fig. 2e, the CC,intraC,intraP component of the RDF from the model and simulation agree very well.

\section{Crystalline-Crystalline, Intracrystallite, Interplanar $(C C$, intraC, interP) Component}

The interaction between two carbon atoms in different planes with the same crystallite is treated at the mesoscale. We assumed each plane was a circular disk with uniform distribution of density. Following the procedure described for the AC, we derived an analytical expression for the interaction between two planes as a function of the distance between them. This distance between planes, $d_{\mathfrak{p}}$, is a parameter in the model. Here we used $3 \mathrm{~A}$, a reasonable value supported by experiment. ${ }^{16} \mathrm{~A}$ nanocrystallite of radius $5 \AA$ contains three planes. Therefore, there will be contributions to the RDF at two distances: adjacent planes and planes separated by one plane between them. As shown in Fig. 2f, the component displays two peaks corresponding to the two distinct distances. Larger crystallites will have more peaks in this component RDF because they contain more planes. At $0 \mathrm{~K}$, these nanoparticles generate a sharp peak. However, at room temperature, the peak due to this planar spacing is broadly distributed, which is why graphitic nanoparticles have been called entropically driven structures. ${ }^{16}$ This feature can be lost in the background of the total RDF, a point that highlights the value of this decomposition.

\section{Weighting Constants}

To the total RDF, each component must be weighted appropriately. These weights are rigorously determined by the relative preponderance of each type of pair correlation, which is a function of the number of atoms in each phase and in each plane. The essential structural quantities to determine these weights are the volume fractions and the densities of the phases, properties that can be provided through other experiments (microscopy and various densometry techniques) or fit.

\section{Total RDF}

The total RDF is shown in Fig. 3. The RDF from simulation and model are obtained by summing the six terms in Fig. 2. The experimental RDF was obtained on the NOMAD beam line at SNS. Careful comparison of the simulated and experimental RDFs has been previously presented. What is

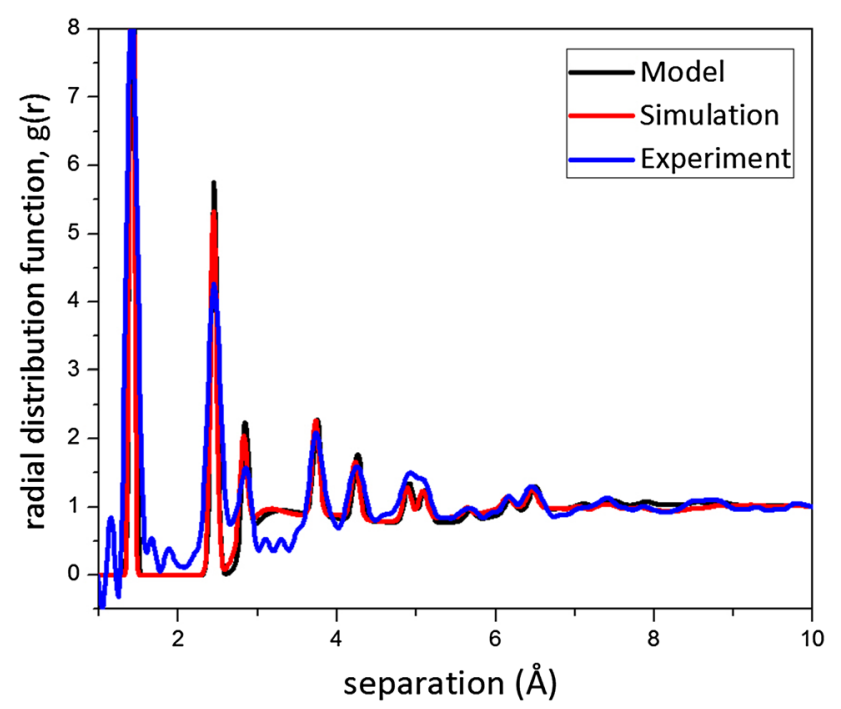

Fig. 3. Comparison of the total RDF from the model to results from MD simulation and NS experiments.

new here is the addition of the model. There is some noise at low separations in the experimental data. Every peak that appears in the experimental data also appears in the simulated and model data; these peaks originate from intraplanar interactions in the amorphous and crystalline phases. The largest observable difference between the simulation and the experimental result appears between the third and fourth peaks at a value slightly greater than $3 \AA$. Perhaps, we have underestimated the fluidity of the planes within such small crystallites, as does the simulation. Regardless, the model offers just as good of a comparison with experiment as does the MD simulation.

The MD simulations of the carbon composite materials included from $70,000 \mathrm{C}$ atoms to 550,000 $\mathrm{C}$ atoms and used $1000 \mathrm{CPU}$ hours to $10,000 \mathrm{CPU}$ hours each. ${ }^{7}$ The model used less than one second of CPU time on a laptop. Thus, for a comparable level of accuracy, the model offers at least six orders of magnitude increase in efficiency. The true value of the simulation was in providing a well-founded basis for the decomposition. Because of its low computational requirement, the model can be used to explore other structural variables in the system, such as polydispersity of nanoparticles or nanoparticle arrangements in a reasonable time frame. Thus, the efficiency gain enables a much broader scientific investigation.

The ultimate goal is to replace computationally expensive MD simulations with theoretical approximations to the greatest extent possible. The interpretation of experimental data could be done in the absence of the decomposition from MD. The parameters in the theory would be fit to the total RDF available from experiment. Each component of the $\mathrm{RDF}$ would be evaluated but the difference between the total RDF generated from experiment and the 
model would serve as the objective function upon which optimization is based. Certainly the model parameters, such as volume fraction of the crystalline phase and size of the nanocrystallites can be initialized and or bounded based on corroborating experimental evidence from electron microscopy. One can also imagine that the presence of a decomposition of the RDF from MD for one example of the material might prove beneficial, while a fuller range of structures due to variation in, for example, a processing parameter is investigated strictly using the more computationally efficient model.

\section{CONCLUSION}

A hierarchical decomposition of the RDF was presented. The process decomposes contributions to the RDF in a hierarchical manner until all components are able to be rapidly evaluated by theory or model, at either the atomic scale or the mesoscale. The model rivals the MD simulation in the ability to explain scattering data of hierarchical materials at a fraction of the computational cost. The hierarchical decomposition of the RDF described earlier and applied to a carbon composite is generalizable and can readily be applied to any composite material in which nanoparticles of one phase are distributed in a matrix of another phase. We anticipate that the model, combined with experiment and simulation, will provide structure-property relationships that will lay the foundation for the discovery of new magnets that can be used in electronics, wind turbines, and electric vehicle motors. This combination of theory, experiment, and simulation is consistent with the aims of the Materials Genome Initiative $^{17}$ for the rapid development of material structures, through high-throughput computational analysis to extract maximum insight from limited experiments.

\section{ACKNOWLEDGEMENTS}

N.M. was supported by a grant from the Oak Ridge Associated Universities High Performance Computing Program and by a Grant from the National Science Foundation (DGE-0801470). This research Project used resources of the National
Institute for Computational Sciences (NICS) supported by NSF under Agreement Number: OCI 07-11134.5. The research at Oak Ridge National Laboratory's Spallation Neutron Source was sponsored by the U.S. Department of Energy, Office of Basic Energy Sciences. Also, part of the research was sponsored by the Critical Materials Institute, an Energy Innovation Hub funded by the U.S. Department of Energy, Office of Energy Efficiency and Renewable Energy.

\section{REFERENCES}

1. A.C. Larson and R.B. Von Dreele, GSAS, General Structure Analysis System (Los Alamos, NM: LANSCE, 1994), pp. 125-176.

2. B.H. Toby, J. Appl. Crystallogr. 34, 210 (2001).

3. S.J.L. Billinge and T. Egami, Underneath the Bragg Peaks, Volume 16, Second Edition: Structural Analysis of Complex Materials (Oxford, U.K.: Pergamon Materials Series, 2013), pp. 55-95.

4. T.E. Proffen and R.B. Neder, Diffuse Scattering and Defect Structure Simulations: A Cook Book Using the Program DISCUS (Oxford, U.K.: Oxford University Press, 2009), pp. 175-201.

5. P.-W. Ma, W.C. Liu, C.H. Woo, and S.L. Dudarev, J. Appl. Phys. 101, 073908 (2007).

6. W.E. Tenhaeff, O. Rios, K. More, and M.A. McGuire, Adv. Funct. Mater. 24, 86 (2014).

7. N.W. McNutt, O. Rios, M. Feygenson, T.E. Proffen, and D.J. Keffer, Appl. Crystallogr. 47, 1577 (2014).

8. L. Petridis, S.V. Pingali, V. Urban, W.T. Heller, H.M. O'Neill, M. Foston, A. Ragauskas, and J.C. Smith, Phys. Rev. E 83, 061911 (2011).

9. M.A. McGuire, O. Rios, N.J. Ghimire, and M. Koehler, Appl. Phys. Lett. 101, 202401 (2012).

10. H.J. Snaith, J. Phys. Chem. Lett. 4, 3623 (2013).

11. M. Gerard, A. Chaubey, and B.D. Malhotra, Biosens. Bioelectron. 17, 345 (2002).

12. R.C. Agrawal and G.P. Pandey, J. Phys. D Appl. Phys. 41, 223001 (2008).

13. S. Chand, J. Mater. Sci. 35, 1303 (2000).

14. Y. Li, C. Pruitt, O. Rios, L. Wei, M. Rock, J.K. Keum, A.G. McDonald, and M.R. Kessler, Macromolecules 48, 2864 (2015).

15. T. Proffen, S.J.L. Billinge, T. Egami, and D. Louca, $Z$. Kristallogr. 218, 132 (2003).

16. N.W. McNutt, Q. Wang, O. Rios, and D.J. Keffer, J. Nanopart. Res. 16, 1 (2014).

17. Materials Genome Initiative for Global Competitiveness (Washington, D.C.: The White House, 2011). www.white house.gov/mgi. 